\title{
A longitudinal investigation of metal accumulation in brain regions of Göttingen minipigs, and a first glimpse of $\mathrm{Ca}$ isotopes in the mammalian brain
}

\author{
B. MAHAN ${ }^{* 1,2}$, M.A. ANTONELLI ${ }^{3,4}$, P. BURCKeL ${ }^{3}$, S.

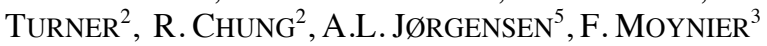 \\ ${ }^{1}$ Earth and Environmental Sciences, James Cook University, \\ Townsville, Queensland 4814, Australia \\ (*correspondence: brandon.mahan@jcu.edu.au) \\ ${ }^{2}$ Thermo Fisher Isotope Development Hub, Department of \\ Earth and Planetary Sciences, Macquarie Unviersity, \\ Sydney, New South Wales 2109, Australia \\ ${ }^{3}$ Université de Paris, Institut de Physique du Globe de Paris, \\ CNRS, 75238 Paris, France \\ ${ }^{4}$ Institute of Geochemistry and Petrology, Department of \\ Earth Sciences, ETH Zürich, 8092 Zürich, Switzerland. \\ ${ }^{5}$ Department of Biomedicine, Aarhus University, Aarhus, \\ Denmark.
}

The metallome is fundamentally important to the biological sphere, and a wealth of evidence suggests that metals are inherent to the mechanisms of neurodegenerative diseases and/or their pathological manifestations. A prominent example of this is the neuronal/brain accumulation of metals such as $\mathrm{Fe}, \mathrm{Cu}$, and $\mathrm{Ca}$ associated with Alzheimer's Disease (AD). Stable metal isotopes have shown utility in differentiating healthy vs. diseased states, sometimes prior to traditional diagnoses, highlighting their potential as early diagnostics. Here we have mapped out the distribution of numerous biologically relevant elements ( $\mathrm{Mg}, \mathrm{P}, \mathrm{K}, \mathrm{Ca}, \mathrm{Fe}, \mathrm{Cu}$, and $\mathrm{Zn}$ ) in brain regions of Göttingen minipigs ranging in age from three months to nearly six years, including wild type, single- and double-transgenic models of $A D$ (PS1, APP/PS1). Preliminary results track tell-tale rises in brain metals with age, namely $\mathrm{Fe}$ and $\mathrm{Cu}$. Moreover, we have characterized $\mathrm{Ca}$ isotopes in the mammalian brain for the first time, and find that the brain represents the most isotopically depleted $\mathrm{Ca}$ reservoir in the body currently known, with $\delta^{44} \mathrm{Ca}$ consistently $\sim 1.5 \%$ lighter than the liver. These results suggest that our efficient $\mathrm{Ca}$ purification and isotopic analysis techniques are robust and can provide critical information on $\mathrm{Ca}$ homeostasis in future studies. 